Revista Verde de Agroecologia e Desenvolvimento Sustentável

http://www.gvaa.com.br/revista/index.php/RVADS

ARTIGO CIENTÍFICO

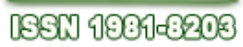

DOI: http://dx.doi.org/10.18378/rvads.v10i3.3585

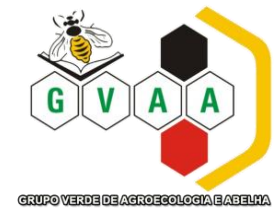

\title{
Elaboração e caracterização de doce de coco adicionado de diferentes concentrações de rapadura
}

\section{Preparation and characterization of doce de coco added to different concentrations of sugar cane products}

\author{
Luan Pedro Melo Azerêdo ${ }^{1 *}$, Heloisa Maria Almeida Do Nascimento ${ }^{1}$, Ricardo Targino Moreira ${ }^{2}$, Emmanuel Moreira \\ Pereira $^{3}$
}

\begin{abstract}
Resumo: A rapadura é o produto sólido obtido da concentração do caldo da cana-de-açúcar, muito procurada pelos consumidores, sendo sua utilização na elaboração de doces uma boa alternativa econômica para seu aproveitamento. Este trabalho teve como objetivo avaliar a qualidade microbiológica e sensorial de três formulações de doce de coco. Os resultados mostram que a formulação mais aceita foi obtida a partir da concentração média de rapadura, contribuindo para o desenvolvimento do doce a base de rapadura por meio de procedimentos simples e de fácil aplicação pelos produtores.
\end{abstract}

Palavras-chaves: Rapadura; produto regional; aceitabilidade.

\begin{abstract}
The brown sugar is the solid product obtained by concentration of the juice of cane sugar, very popular with consumers, and is used to manufacture candy good economic alternatives for their utilization. This study aimed to evaluate the microbiological and sensory quality of three sweet coconut formulations. The results show that the most accepted formulation was obtained from the average concentration of brown sugar, contributing to the development of the sweet brown sugar base through simple procedures and easy to apply by producers.
\end{abstract}

Key words: Brown Sugar; regional product; acceptability.

\footnotetext{
*Autor para correspondência

Recebido para publicação em 25/05/2015; aprovado em 20/07/2015

${ }^{1}$ Programa de Pós-Graduação em Ciências e Tecnologia de Alimentos - Curso de Mestrado - Universidade Federal da Paraíba - UFPB- 58051-900- João

Pessoa - PB - Brasil. E-mail: heloysa28@gmail.com, luan_p22@hotmail.com

${ }^{2}$ Porfessor UFPB, Departamento de Tecnologia Quimíca e de Alimentos DTQA, E-mail: ricardo.ufpb@ gmail.com

${ }^{3}$ Pós-graduando em Engenharia Agrícola - UFCG/CTRN - Universidade Federal de Campina Grande-PB, e-mail: emmanuel16mop@ hotmail.com
} 


\section{INTRODUÇÃO}

No Brasil, a cana foi introduzida pelos colonizadores portugueses, provenientes da Ilha da Madeira, impulsionando a formação dos primeiros engenhos no país sendo responsáveis pelo desenvolvimento da produção, do comércio e da cultura do Nordeste brasileiro (UNICA, 2004). A canade-açúcar (Saccharum spp.) é considerada uma das melhores opções dentre as fontes de energia renováveis, com grande importância no cenário agrícola brasileiro (SOUZA et al., 2005), sendo considerada uma matéria-prima de aproveitamento quase integral, já que seus subprodutos e resíduos podem ser utilizados na alimentação animal e humana (ALCARDE, 2007).

A cana-de-açúcar quando processada gera vários produtos, entre eles encontra-se a rapadura. Ela é amplamente apreciada pela população nordestina, que além de contribuir com seu sabor e qualidades nutricionais, é uma importante fonte de emprego e renda à população (SOUZA et al., 2005). Em termos de mercado, a rapadura ainda encontra alguns entraves, já que o mercado consumidor é pouco exigente, dificultando a utilização da rapadura como ingrediente no desenvolvimento de produtos alimentícios. A rapadura é um produto sólido a temperatura ambiente, de sabor doce, obtido pela concentração a quente do caldo da cana-de-açúcar, sua principal matéria-prima, sendo o seu ponto final conseguido por desidratação do caldo em torno de $92^{\circ}$ Brix. Ela tem sabor e odor agradável e característico, além de elevado valor alimentício (LUI et al., 2011). Em geral, as rapaduras são quase inteiramente compostas de açúcares (sacarose, principalmente), e de uma pequena quantidade de outros componentes (água, cinzas, proteínas e sólidos insolúveis). Além disso, a presença de um importante grupo de minerais (K, $\mathrm{Ca}, \mathrm{P}, \mathrm{Mg}, \mathrm{Na}, \mathrm{Fe}, \mathrm{Mn}, \mathrm{Zn}$ e $\mathrm{Cu}$ ) foi comprovada, o que aumenta os benefícios nutricionais da rapadura (GUERRA \& MUJICA, 2010).

O desenvolvimento de novas apresentações é uma das estratégias para promover o consumo de Rapadura. Devido à sua elevada proporção de sacarose e as vantagens já mencionadas, ele pode ser usado da mesma forma como o açúcar (bebidas, sobremesas e preparações doce) (GUERRA \& MUJICA, 2010). A utilização da rapadura em doces de frutas é uma prática pouco explorada pelos pesquisadores, com isso visando utilizar esse produto típico da região nordeste na confecção de novos produtos, esse trabalho teve como objetivos elaborar três formulações de doce de coco utilizando diferentes concentrações de rapadura, para com isso avaliar a qualidade microbiológica e sensorial desses doces.

\section{MATERIAL E MÉTODOS}

\section{Materiais}

Os cocos secos (Cocos nucífera L. var. Typica) utilizados para a elaboração dos doces foram adquiridos no comércio local de João Pessoa/PB. As rapaduras, utilizadas para a elaboração do doce, também foram adquiridas no comércio da cidade de João Pessoa-PB, provenientes do engenho Bujari, localizado no município de Areia-PB.

\section{Processamento dos Doces}

No fluxograma exibido pela figura 1, estão expostas as etapas do processamento do doce de coco.

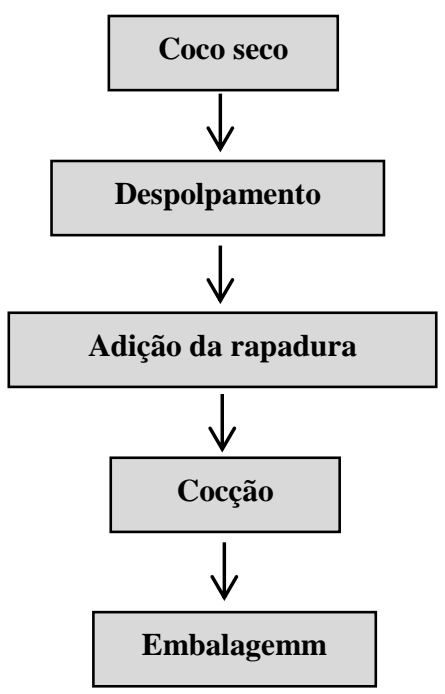

Figura 1. Fluxograma de elaboração do doce de coco.

Foram elaboradas três formulações de doce de coco com diferentes concentrações de rapadura, conforme Tabela 1. Para elaboração foi utilizado coco seco, rapadura, ácido cítrico (suco de limão) e cravo da índia. O doce foi preparado em tacho de aço inoxidável, logo após a adição da polpa do coco e da rapadura em concentrações distintas. $\mathrm{O}$ ácido cítrico foi incorporado no doce ao final do processo de cocção. Após o término da cocção, os doces foram armazenados ainda quente em recipientes de polipropileno, resfriados à temperatura ambiente, e armazenados sob refrigeração, até o momento das análises.

Tabela 1 - Formulações dos doces

\begin{tabular}{cccc} 
Formulações & Coco $(\mathbf{g})$ & Rapadura $(\mathbf{g})$ & $\mathbf{( \% )}$ \\
\hline T 1 & 250 & 400 & 44 \\
T 2 & 250 & 300 & 55 \\
T 3 & 250 & 200 & 62 \\
\hline
\end{tabular}

\section{Análises Microbiológicas}

As análises microbiológicas foram realizadas, antes da análise sensorial, nas três formulações do doce de coco, através da metodologia descrita por Silva et al, (2007). Os doces foram avaliados quanto à contagem em placas de bolores e leveduras e a enumeração dos coliformes a $35{ }^{0} \mathrm{C}$ e coliformes termotolerantes. A contagem de bolores e leveduras foi realizada através da semeadura em placas contendo o meio Ágar Batata, incubadas a $25{ }^{\circ} \mathrm{C}$ por 72 horas. A detecção de coliformes a $35^{\circ} \mathrm{C}$ e termotolerantes foi realizada através da técnica do Número Mais Provável (NMP) em tubos múltiplos. Para confirmação dos coliformes a $35^{\circ} \mathrm{C}$, foi utilizado Caldo Lactose Bile Verde Brilhante (CLBVB), e para o teste confirmativo de termotolerantes foi utilizado o caldo Escherichia coli (EC), incubados a $45^{\circ} \mathrm{C}$ por 24 horas. 


\section{Análise Sensorial}

A análise sensorial dos doces foi realizada no laboratório de análise sensorial do Departamento de Engenharia de Alimentos da UFPB, em cabines individuais próprias para testes sensoriais, com iluminação artificial uniformemente distribuída, longe de ruídos e odores, em horários previamente estabelecidos, excluindo $1 \mathrm{~h}$ antes e $2 \mathrm{~h}$ após o almoço. As amostras de doces foram servidas em temperatura ambiente, em porções de aproximadamente $5 \mathrm{~g}$, apresentadas em copos plásticos descartáveis $(50 \mathrm{~mL})$ codificados e acompanhados de biscoito tipo Cream Cracker e de água, e da ficha de avaliação. Utilizou-se um grupo de 50 provadores não treinados, sendo $56 \%$ do gênero feminino e $44 \%$ do gênero masculino, os quais receberam orientações específicas sobre os testes antes da realização.

Todos os provadores receberam e responderam um questionário antes da análise sensorial, para quantificar os provadores que possui o habito de consumir doces, rapaduras e de produtos que possuam a rapadura na sua formulação.

Os atributos sensoriais analisados foram: aroma, cor, sabor, textura e aceitação global. Para avaliar esses atributos, os provadores receberam uma ficha contendo uma escala hedônica estruturada de nove pontos, variando de 9- gostei extremamente a 1- desgostei extremamente (CHAVES \& SPROESSER, 1999). Na mesma ficha os provadores receberam uma escala para expressar a intenção de consumo, contendo cinco pontos, variando de 5- certamente consumiria a 1- certamente não consumiria.

\section{Análise Estatística}

Os resultados da análise sensorial foram submetidos à análise de variância (ANOVA) para a obtenção das médias, e essas foram comparadas pelo teste de Turkey a 5\% de significância a fim de observar se houve diferença significativa entre os tratamentos. Os resultados estatísticos foram tratados pelo programa $\mathrm{SAS} \circledast(\mathrm{SAS}, 1999)$.

\section{RESULTADOS}

A figura 2 mostra a frequência de consumo de doces por parte dos provadores participantes da análise sensorial.

Figura 2. Consumo de doces de frutas.
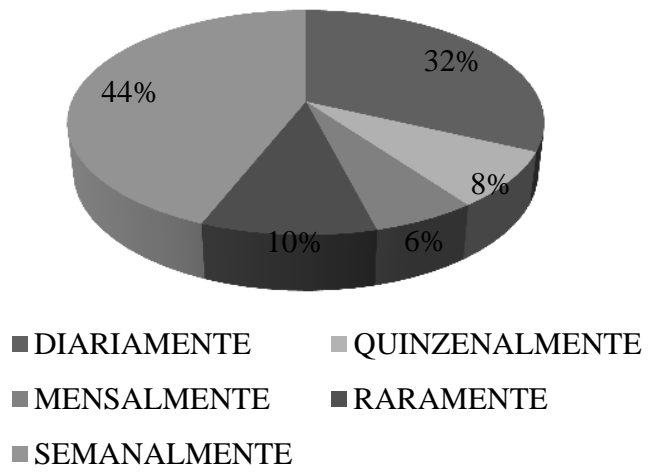

A maioria dos provadores, participantes da análise sensorial, consomem doces de frutas semanalmente ou diariamente, já que os percentuais de consumo semanalmente e diariamente foram $44 \%$ e $32 \%$, respectivamente.

Figura 3. Levantamento dos provadores que consomem rapadura.

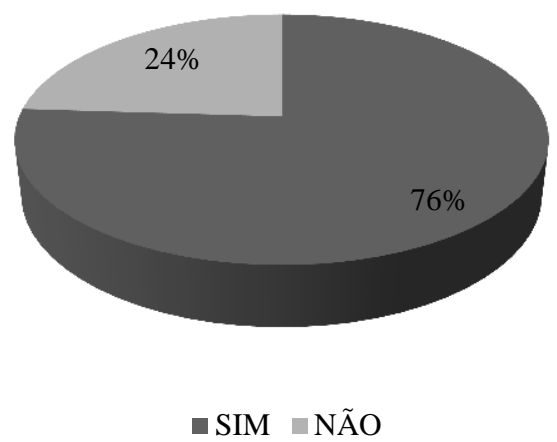

A figura 3 mostra que $24 \%$ dos provadores não consomem rapadura, sendo esse um número alto considerando que a rapadura é um produto típico da região nordeste, principalmente da Paraíba. Esse fato pode ser reflexo de hábitos alimentares e do número reduzido de produtos, disponíveis no mercado, que possuem a rapadura na sua composição.

A figura 4 mostra a frequência do consumo de rapadura pelos provadores que afirmaram consumir. Com isso é possível observar que mesmo entre os consumidores de rapadura, a frequência de consumo diário desse derivado da cana-de-açúcar é muito baixa.

Figura 4. Frequência de consumo de rapadura pelos provadores.

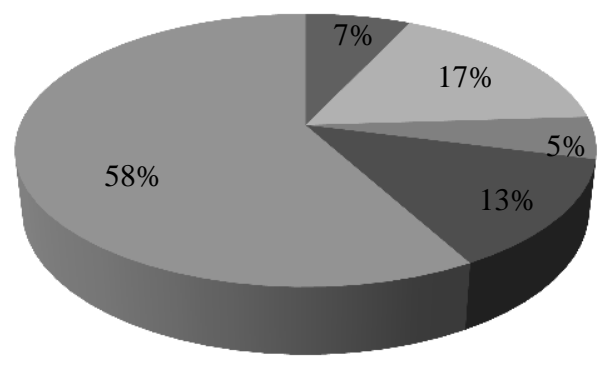

- Diario $\square$ semanal $\square$ Quinzenal $\square$ Mensal $\square$ Raramente

Antes da realização das análises sensoriais as amostras foram submetidas à análise microbiológica originando os resultados expostos na tabela 2 . 
Tabela 2 - Análise microbiológica de doce de coco com diferentes concentrações de rapadura.

\begin{tabular}{cccc}
\hline Tratamentos & $\begin{array}{c}\text { Coliformes Totais } \\
\left(\mathrm{NMP} \cdot \mathrm{g}^{-1}\right)\end{array}$ & $\begin{array}{c}\text { Coliformes Termotolerantes } \\
\left(\mathrm{NMP} \cdot \mathrm{g}^{-1}\right)\end{array}$ & $\begin{array}{c}\text { Bolores Leveduras } \\
\left(\mathrm{UFC}^{-1} \mathrm{~g}^{-1}\right)\end{array}$ \\
\hline T1 & $<3$ & $<3$ & $<10^{*}$ \\
$\mathrm{~T} 2$ & $<3$ & $<3$ & $<10^{*}$ \\
T3 & $<3$ & $<3$ & $<10^{*}$ \\
\hline
\end{tabular}

\section{*Estimado}

As amostras analisadas apresentaram o Número Mais Provável (NMP) <3, indicando que não houve presença de coliformes totais e termotolerantes. De acordo com a legislação $\mathrm{n}^{\circ} 12$ de janeiro de 2001 (BRASIL, 2001), a tolerância de bolores e leveduras para doce em pasta é de $10^{4}$ UFC (Unidades Formadoras de Colônias), o que não foi encontrado em nenhuma das amostras analisadas. Diante desses resultados as amostras se mostraram aptas para o consumo.

$\mathrm{Na}$ figura 5 é encontrado o perfil sensorial dos provadores em relação às amostras de doce de coco elaborados com três concentrações distintas de rapadura.

Figura 5. Perfil da aceitação dos doces de coco adicionados de diferentes concentrações de rapadura

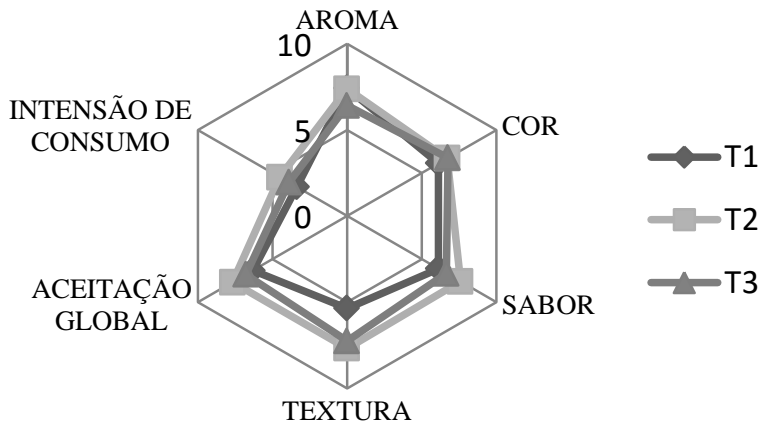

Na figura 5 é possível observar que T2 obteve as maiores notas para todos os atributos sensoriais avaliados. O tratamento T1, só recebeu as maiores notas para o aroma, pois nos demais atributos esse tratamento recebeu notas inferiores aos demais. O tratamento $\mathrm{T} 3$, assim como $\mathrm{T} 2$ obtiveram as maiores notas quando avaliado o atributo cor.

No sabor, percebeu-se que não houve diferença significativa entre os tratamentos T1 e T3, mas eles diferiram significativamente em relação ao T2, o qual obtive a maior média, isso mostra que a concentração de rapadura utilizada no tratamento 2 foi mais aceita em relação as demais, possuindo uma palatabilidade melhor.

Na tabela 3 é possível observar o índice de aceitação dos doces

Tabela 3. Índice de aceitação para os doces de coco

\begin{tabular}{cc}
\hline Tratamentos & Índice de Aceitação $(\%)$ \\
\hline T1 & 70,22 \\
T2 & 85,33 \\
T3 & 75,33 \\
\hline
\end{tabular}

Os resultados para o índice de aceitação do tratamento 2 estão de acordo com os resultados encontrados por Santana \& Oliveira (2005), que obteve índice de aceitabilidade de $85,33 \%$ (tabela 4 ).

Na tabela 4 estão apresentadas as médias das notas para os atributos sensoriais e intenção de consumo das três formulações de doces de coco, que foram obtidas na análise sensorial. Os resultados expressos na tabela 3 permitem observar que não houve diferença significativa entre $(p \leq 0,05)$ entre os três tratamentos, para o atributo cor, já para os atributos aroma, sabor, textura e aceitação global os tratamentos apresentaram diferença significativa.

Para o atributo aroma não foi observado diferença entre os tratamentos $\mathrm{T} 1$ e $\mathrm{T} 2$, onde esses obtiveram valores médios superiores ao tratamento T3, diferindo significativamente. Provavelmente o aroma característico de rapadura foi mais pronunciado nos tratamentos com maior concentração (T1 e T2), sendo esse um dos critérios de aceitação do produto.

Quanto à aceitação global, o tratamento T2 diferiu significativamente dos demais, obtendo a melhor média $(7,68)$ que corresponde a gostei moderadamente na escala hedônica de nove pontos. As médias dos demais tratamentos (T1: 6,32 e T3: 6,70) correspondem à nota gostei ligeiramente, indicando que também foram bem aceitos pelos provadores.

Tabela 4 - Médias das notas obtidas para cada atributo sensorial dos doces de coco.

\begin{tabular}{cccccc}
\hline & \multicolumn{5}{c}{ Atributos } \\
\cline { 2 - 6 } Formulações & Aroma & Cor & Sabor & Textura & Aceitação global \\
\hline T1 & $7,42 \mathrm{a}$ & $6,12 \mathrm{a}$ & $6,12 \mathrm{~b}$ & $5,36 \mathrm{~b}$ & $6,32 \mathrm{~b}$ \\
T2 & $7,38 \mathrm{a}$ & $6,72 \mathrm{a}$ & $7,60 \mathrm{a}$ & $7,68 \mathrm{a}$ & $7,68^{\mathrm{a}}$ \\
T3 & $6,42 \mathrm{~b}$ & $6,76 \mathrm{a}$ & $6,66 \mathrm{~b}$ & $7,26 \mathrm{a}$ & $6,70 \mathrm{~b}$ \\
\hline
\end{tabular}

*As médias acompanhadas com as mesmas letras não diferem entre si (Teste de Turkey a 5\% de probabilidade). 
$\mathrm{Na}$ figura 6 estão presentes os valores médios da intenção de consumo para cada tratamento analisado.

Figura 6. Intenção de consumo do doce de coco.

T3

$\mathrm{T} 2$

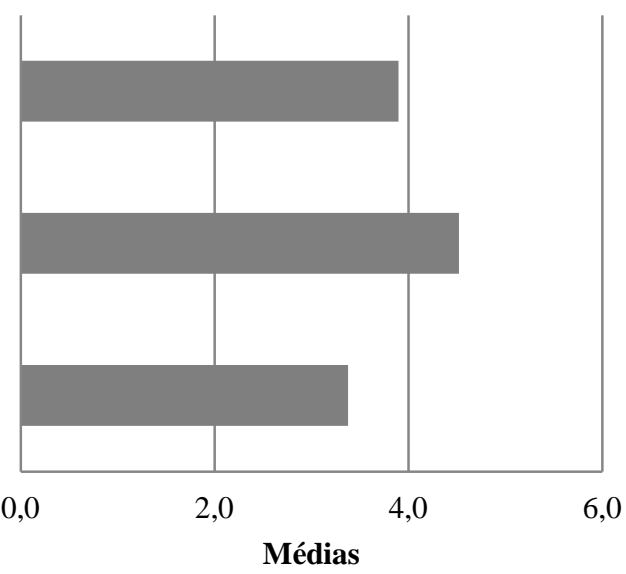

$\mathrm{O}$ tratamento $\mathrm{T} 2$ apresentou a maior intenção de consumo pelos provadores, como pode ser observado na figura 6. Esse resultado confirma os dados encontrados na análise de aceitação global, a qual mostra uma preferência dos provadores pelo doce elaborado com concentração intermediária $(55 \%)$ de rapadura.

\section{CONCLUSÕES}

Diante dos resultados expostos a partir das análises sensoriais, o doce elaborado com $55 \%$ de rapadura foi o que apresentou as melhores médias em todos os atributos sensoriais analisados. Os procedimentos artesanais e as formulações que foram aplicados na elaboração dos doces de coco mostram-se pertinentes, pois proporcionaram qualidades sensoriais ao produto, de forma a permitir aplicabilidade para os pequenos e médios produtores de rapadura. Os doces foram sensorialmente aceitos pelos provadores. Contribuindo para o desenvolvimento e possível inserção de novos produtos utilizando a rapadura como fonte energética e de nutrientes.

\section{REFERÊNCIAS BIBLIOGRÁFICAS}

ALCADE, A. R. Processamento da cana-de-açúcar. Disponível

<www.agencia.cnptia.embrapa.br/gestor/cana-deacucar/arvore>. Acesso em: 02 de outubro de 2011.

Sanitária. Resolução Normativa $n^{\circ} 9$ de 1978. Diário Oficial da União, Poder Executivo. Brasília, 1978.

BRASIL. Agência Nacional de Vigilância Sanitária. Resolução de Diretoria Colegiada - RDC no 12, de 2 de janeiro de 2001. Diário Oficial da União, Poder Executivo. Brasília, 2001.
BRASIL. Agência Nacional de Vigilância Sanitária. Resolução de Diretoria Colegiada - RDC no 271, de 22 de setembro de 2005. Diário Oficial da União, Poder Executivo. Brasília, 2005.

BRASIL. Ministério da Agricultura, Pecuária e Abastecimento. Cana-de-açúcar. Disponível em: http://www.agricultura.gov.br/vegetal/culturas/cana-deacucar. Acesso em: 02 de nov. de 2011.

CARDOSO, J. R.; SILVA, F. I. C.; BRAGA, L. O.; MORAES, G. B. de; LIMA, A. de. Avaliação da aceitabilidade de doce de casca de mamão com coco e doce de polpa de mamão com coco. Anais do Congresso Norte-Nordeste de Pesquisa e Inovação. Maceió-AL, 2010.

CHAVES, J. B. P.; SPROESSER, R.L. Práticas de laboratório de análise sensorial de alimentos e bebidas. Viçosa: Editora UFV, 1999.

GUERRA, M. J. \& MUJICA, M. V. Physical and chemical properties of granulated cane sugar "panelas". Ciência Tecnologia de Alimentos, vol.30, n.1, pp. 250-257. Epub Mar 05, 2010.

LUI, J. J.; FIDELIS, R. R.; DIAS, M. A. R.; SARMENTO, R. A. Produtividade de rapadura de genótipos de cana-deaçúcar na Região de Dueré, Sul de Tocantins. Semina: Ciências Agrárias, Londrina, v. 32, n. 3, p. 1059-1068, jul/set. 2011.

SANTANA, A. F.; OLIVEIRA, L. F. de. Aproveitamento da casca de melancia (Curcubita citrullus, Shrad) na produção artesanal de doces alternativos. Alimentos e Nutrição. v. 16, p.363-368, 2005.

SILVA, N. et al. Manual de Métodos de Análise Microbiológica de Alimentos. São Paulo: Livraria Varela, 2007.

SOUZA, C. C. de; DANTAS, J. P.; SILVA, S. de M.; SOUZA, V. C. de; ALMEIDA, F. A. de; SILVA, L. E. da. Produtividade do sorgo granífero cv. sacarino e qualidade de produtos formulados isoladamente ou combinados ao caldo de cana-de-açúcar. Ciência Tecnologia de Alimentos. n.25, p.512-517, 2005a.

SOUZA, Z. M. de; PAIXÃO, A. C. S.; PRADO, R. de M.; CESARIN, L. G.; SOUZA, S. R. de. Manejo de palha de cana colhida sem queima, produtividade do canavial e qualidade do caldo. Ciência Rural, v.35, n5, 2005 b.

UNICA. Cana-de-açúcar: história. Disponível em: http://www.unica.com.br/content. 2004. Acesso em: 30 setembro 2011. 